\title{
Antioxidant Screening on the Whole Plant of Cyanthillium cinereum (L) H.Rob
}

\author{
Mohammed Shihab K. K. ${ }^{1}$, Rajagopal P. L. ${ }^{2}$, Nasila K. ${ }^{3}$, Neethu Krishnan S. , \\ Harsha CT. ${ }^{5}$ \\ ${ }^{1,3,4,5}$ Final Semester, M.Pharm Student, College of Pharmaceutical Sciences, Government Medical College, \\ ${ }^{2}$ Professor of Pharmacognosy, College of Pharmaceutical Sciences, Government Medical College, Kannur,
} Kerala.

Corresponding Author: Mohammed Shihab K.K.

\begin{abstract}
Methanolic extract of the whole plant of Cyanthillium cinereum (L) H.Rob was evaluated for free radical scavenging activity by using Nitric oxide scavenging activity (NO) and Reduction of 1, 1-Diphenyl -2-Picryl Hydrazyl (DPPH) free radical scavenging activity. Throughout the studies the plant extract showed potent antioxidant activity and the activity was found to be concentration dependent which may be attributed to the high flavonoid content of the plant.
\end{abstract}

Keywords- Cyanthillium cinereum (L) H.Rob, $\mathrm{DPPH}, \mathrm{NO}$

\section{INTRODUCTION}

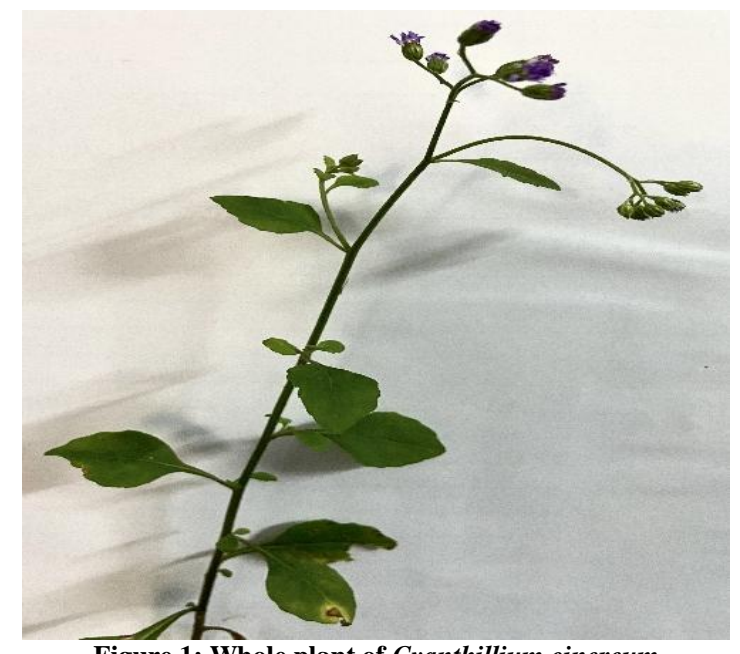

Figure 1: Whole plant of Cyanthillium cinereum

Cyanthillium cinereum (L.) H.Rob. (Asteraceae) is also known as Sahadevi in Sanskrit. It is an erect, rarely decumbent, branched herb, $12-75 \mathrm{~cm}$ high, found throughout India ascending to an altitude of $1800 \mathrm{~m}$. The whole plant of Cyanthillium cinereum is shown in Figure 1. The plant has reputation as folklore medicine in various traditional systems of medicine ${ }^{[1]}$. The plant is used as an analgesic, antimicrobial, antipyretic and antiinflammatory agent ${ }^{[2]}$. The plant contains flavonoids, glycosides, alkaloids, triterpenoids and other phenolic constituents [3].

Oxidation is a normal and necessary process that takes place in our body. Oxidative stress, on the other hand, occurs when there's an imbalance between free radical activity and antioxidant activity ${ }^{[4]}$. Antioxidants are substances that generally prevent the process of oxidation, which produce free radicals, even at a very low concentration. Free radicals are either highly reactive atom or molecules with unpaired electron which can trigger a chain reaction, successively producing more free radicals, leading to eventual death of cells and tissues. Antioxidants are also known as free radical scavengers or reducing agents and it converts these radicals into less reactive species ${ }^{[5]}$.

\section{MATERIALS AND METHOD}

\subsection{Plant material and Preparation of the} extract

The fresh whole plant of Cyanthillium cinereum (L) H. Rob was 
collected from the Government medical college campus Kannur, Kerala. The plant material was identified and a voucher specimen bearing number CC-MS/01/21 was deposited in the department of Pharmacognosy, College of pharmaceutical sciences, Government medical college, Kannur, Kerala. The plant was dried and powdered mechanically and stored in an air tight container. The extraction was carried out by hot percolation method. The solvent used was methanol. The extract was concentrated to dryness under controlled temperature.

\subsection{DPPH scavenging activity}

DPPH is chemically 2,2-diphenyl-1picrylhydrazyl. This assay works on the principle of reduction of DPPH by antioxidant, which is spectrophotometrically measured. This method is the most widely used in-vitro antioxidant assay method. DPPH is a stable free radical with violet color which gets reduced in the presence of a substrate which can donate a hydrogen atom and becomes colorless. To determine the antioxidant activity, the test extract diluted with methanol is added with DPPH solution and after 30 minutes of the procedure, the absorbance values measured at $517 \mathrm{~nm}$. A reduction in the absorbance indicates significant antioxidant activity. Percentage DPPH scavenging is calculated using the following equation where, $A_{b}$ is the absorbance of solution before the reaction and $A_{a}$ is the absorbance of solution after the reaction ${ }^{[6]}$.

$\%$ inhibition of DPPH radical $=\left(A_{b}-A_{a} /\right.$ A $) \times 100$

\subsection{Nitric oxide scavenging activity}

In aqueous medium, sodium nitroprusside generates nitric oxide at physiological $\mathrm{pH}$ (7.2), which is very unstable and hence it reacts with oxygen and produce nitric ions. This nitric ions can be estimated with the help of Griess reagent. Substance to be tested acts as a scavenger of this nitric oxide by competing with oxygen and therefore the amount of the ions produced will decrease substantially. Percentage scavenging of nitric oxide by both the standard and the test substance are assessed spectrophotometrically from their absorbance values and it is calculated and compared. Absorbance of both the test and standard solutions are measured at $540 \mathrm{~nm}$ [7].

\section{RESULTS}

Table 1: Effect of alcoholic extract of the whole plant of Cyanthillium cinereum on DPPH scavenging.

\begin{tabular}{|l|l|l|l|l|l|}
\hline \multirow{2}{*}{ Sl.No. } & \multirow{2}{*}{$\begin{array}{c}\text { Conc. } \\
\end{array}$} & \multicolumn{1}{|c|}{ Alcoholic extract } & \multicolumn{2}{|l|}{ Ascorbic acid (Standard) } \\
\cline { 3 - 6 } & $\mathbf{A b s}$ & \% Sca. & Abs & \%ca. \\
\hline 1 & 5 & 0.979 & 2.51 & 0.937 & 12.39 \\
\hline 2 & 10 & 0.874 & 11.64 & 0.677 & 29.44 \\
\hline 3 & 15 & 0.801 & 19.37 & 0.594 & 49.27 \\
\hline 4 & 25 & 0.769 & 28.69 & 0.492 & 61.70 \\
\hline 5 & 50 & 0.694 & 37.11 & 0.086 & 69.99 \\
\hline 6 & 100 & 0.592 & 52.65 & 0.069 & 75.88 \\
\hline 7 & 250 & 0.327 & 69.17 & 0.055 & 87.64 \\
\hline 8 & 500 & 0.299 & 74.33 & 0.039 & 91.29 \\
\hline 9 & 1000 & 0.117 & 98.55 & 0.021 & 99.70 \\
\hline 10 & Control & 0.819 & & 0.877 & \\
\hline
\end{tabular}

Table 2: Effect of alcoholic extract of the whole plant of Cyanthillium cinereum on NO scavenging.

\begin{tabular}{|l|l|l|l|l|l|}
\hline \multirow{2}{*}{ Sl.No. } & \multirow{2}{*}{$\begin{array}{c}\text { Conc. } \\
\end{array}$} & \multicolumn{2}{|c|}{ Alcoholic extract } & \multicolumn{2}{|c|}{ Ascorbic acid (Standard) } \\
\cline { 3 - 6 } & Abs & \% Sca. & Abs & \% Sca. \\
\hline 1 & 5 & 0.895 & 1.99 & 0.899 & 9.77 \\
\hline 2 & 10 & 0.817 & 2.88 & 0.801 & 19.25 \\
\hline 3 & 15 & 0.633 & 12.14 & 0.727 & 31.60 \\
\hline 4 & 25 & 0.527 & 29.77 & 0.596 & 49.11 \\
\hline 5 & 50 & 0.404 & 38.11 & 0.539 & 57.72 \\
\hline 6 & 100 & 0.391 & 77.24 & 0.396 & 69.32 \\
\hline 7 & 250 & 0.297 & 80.11 & 0.311 & 74.65 \\
\hline 8 & 500 & 0.214 & 89.55 & 0.287 & 90.23 \\
\hline 9 & 1000 & 0.112 & 97.33 & 0.121 & 99.55 \\
\hline 10 & Control & 0.817 & & 0.832 & \\
\hline
\end{tabular}




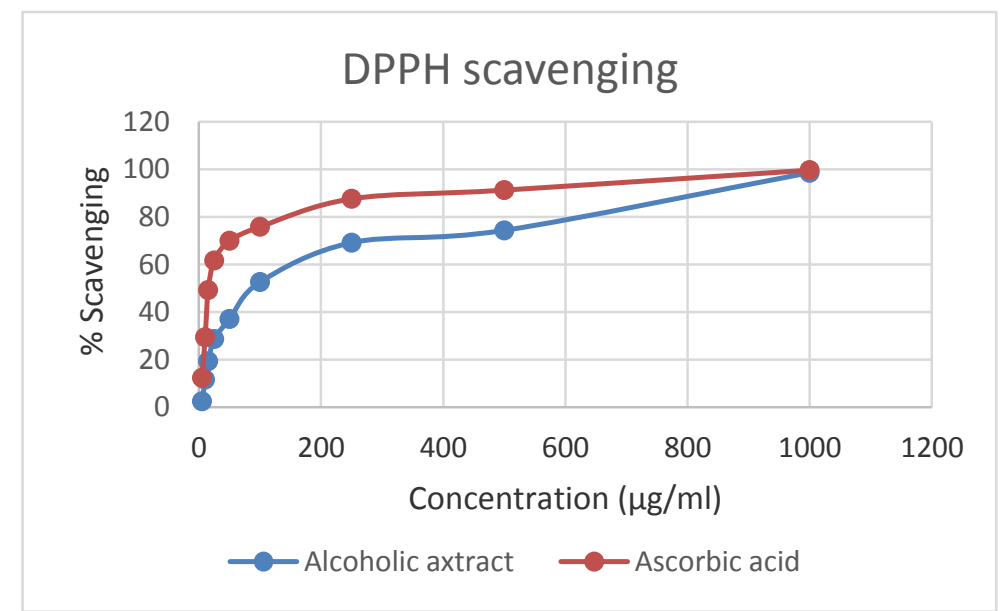

Figure 1: Effect of alcoholic extract of the whole plant of Cyanthillium cinereum on DPPH scavenging.

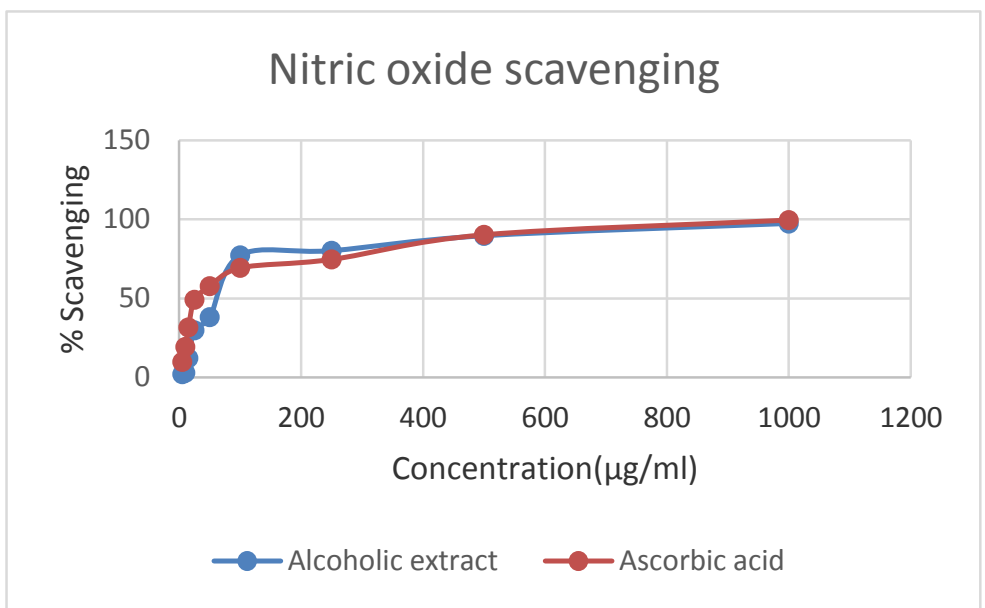

Figure 2: Effect of alcoholic extract of the whole plant of Cyanthillium cinereum on NO scavenging

The alcoholic extract of the whole plant of Cyanthillium cinereum exhibited antioxidant activity in the DPPH and the nitric oxide radical inhibition assay as evidenced by the percentage scavenging which is compared with standard ascorbic acid. (Table 1,2). The graphical comparison of percentage scavenging of alcoholic extract and ascorbic acid versus concentration in DPPH inhibition assay and NO inhibition assay is shown in Figure 1 and Figure 2 respectively.

\section{DISCUSSION}

In our study the methanolic extract of the whole plant of Cyanthillium cinereum showed significant free radical scavenging activity in both the in vitro models like DPPH scavenging and NO scavenging. The preliminary phytochemical screening of the plant extract showed the presence of chief secondary metabolites like alkaloids, flavonoids, poly phenolic compounds, tannins etc. In addition Shruthi Roy et.al, Nishadh et.al reports the presence of above said constituents in the plant extract ${ }^{[3,8]}$. Flavonoids are well documented to have potent antioxidant activity. Polyphenolic compounds and tannins are proven good natural anti-oxidants. Flavonoids have the ability to directly scavenge the reactive oxygen species. They can also chelate free radicals by donating a hydrogen atom or by single-electron transfer. Flavonoids can also act as an intracellular antioxidant through inhibition of free radical generating enzymes like xanthine oxidase, lipoxygenase, protein kinase etc. Phenolic compounds possess redox properties that allow them to act as potent antioxidants. The free radical scavenging ability of phenolic compounds are facilitated by their 
hydroxyl groups hence the total phenolic concentration could be used as a basis for screening of antioxidant activity.

Hence the potent antioxidant potential exhibited by the whole plant of Cyanthillium cinereum could be due to the presence of flavonoids, poly phenolics and tannins.

\section{CONCLUSION}

From the above results the present study was concluded that the alcoholic extract of the whole plant of Cyanthillium cinereum L. H. Rob exhibits anti-oxidant activity in both DPPH radical and NO radical inhibition assays. The anti-oxidant activity exhibited by Cyanthillium cinereum could be due to the presence of flavonoids, poly phenolic compounds and tannins.

\section{ACKNOWLEDGEMENT}

All authors have no conflicts of interest. This research did not receive any specific grant from any funding agency.

\section{Conflict of Interest: None}

\section{Source of Funding: None}

\section{Ethical Approval: Approved}

\section{REFERENCES}

1. Dogra NK, Kumar S. A review on ethnomedicinal uses and pharmacology of Vernonia cinerea Less. Nat Prod Res. 2015;18;(29):12-17.
2. Suresh SN, Varsha V, Prejeena V: Phytochemical screening of Cyanthellium cinereum leaf extracts. International Journal of Medicine and Pharmaceutical Research 2015; 3(6): 1238-1241.

3. Nishadh, Selvi V, Mahalakshmi R. In-Vitro Antioxidant and Phytochemical screening of various extracts of Vernonia cinerea Leaves. Int J Drug Dev Res. 2013; 5(3):227-230.

4. Sharma A, Bhardwaj S, Mann AS, Jain A, Kharya MD. Screening Methods of Antioxidant Activity: An Overview. Pharmacogn Rev 2007; 1 (2):2-5.

5. Mandal S, Yadav S, Neema RK. Antioxidants-a-review. Journal of Chemical and Pharmaceutical Research. 2009; 1(1):102-104.

6. Krishnaiah D, Sarbatly R, Nithyanandam R. A review of the antioxidant potential of medicinal plant species. Food Bioprod Process. 2011; 89(3):217-233.

7. Marcocci L. The nitric oxide-scavenging properties of Ginkgo biloba extract EGB 761. Biochem Biophys Res Commun. 1994; 201:748-755.

8. Shruthi Roy, Madhu KP, Jyolsna G Krishna. Pharmacognostical and phytochemical evaluation of the drug Cyanthillium cinereum. International Journal of Ayurveda and Pharma Research.2019,7(9):19-27.

How to cite this article: Mohammed Shihab K.K., Rajagopal P.L., Nasila K. et.al. Antioxidant screening on the whole plant of Cyanthillium cinereum (L) H.Rob. International Journal of Research and Review. 2021; 8(7): 458-461. DOI: https://doi.org/10.52403/ijrr. 20210764 